\title{
EVALUACIÓN DE LA CALIDAD ESPERMÁTICA EPIDIDIMAL EN HIPOPÓTAMOS HIPPOPOTAMUS AMPHIBIUS (ARTIODACTYLA: HIPPOPOTAMIDAE) UBICADOS EN EL MAGDALENA MEDIO, COLOMBIA
}

\section{GIOvanni RESTREPO BETANCUR, ${ }^{1, *}$ Elizabeth VARELA GIRALDO ${ }^{2}$ y AleXANDra USUGA SUAREZ ${ }^{3}$}

\author{
${ }^{1}$ Universidad Nacional de Colombia, Facultad de Ciencias Agrarias, Calle 59 A No. 63-20, Medellín, Colombia. \\ *Email autor corresponsal: <grestre0@unal.edu.co> \\ ${ }^{2}$ Politécnico Colombiano Jaime Isaza Cadavid, Facultad de Ciencias Agrarias, Carrera 48 No. 7-151, Medellín, \\ Colombia. Email: <elivarelagiraldo@gmail.com> \\ ${ }^{3}$ Universidad CES, Facultad de Medicina Veterinaria y Zootecnia, Calle 10 A No. 22 - 04, Medellín, Antioquia, \\ Colombia. Email: <ausuga@ces.edu.co> \\ Recibido: 30/10/2015; aceptado: 22/04/2016
}

Restrepo, G., Varela, E. \& Usuga, A. 2016. Evaluación de la calidad espermática epididimal en hipopótamos Hippopotamus amphibius (Artiodactyla: Hippopotamidae) ubicados en el Magdalena Medio, Colombia. Acta Zoológica Mexicana (n. s.), 32(2): 158-167.

RESUMEN. El hipopótamo común (Hippopotamus amphibius) es un mamífero del orden Artiodactyla, que habita en África, con una población decreciente a nivel global. Con fines de conservación genética, se han realizado procesos de biotecnología reproductiva, entre los que se reporta, la recuperación de espermatozoides por métodos como la electroeyaculación y la extracción desde epidídimos. Este mamífero es considerado una especie invasora exótica y fue introducido en Colombia en 1985; actualmente se reproduce de forma descontrolada en la región del Magdalena Medio. Como medida de control poblacional, se ha realizado la esterilización quirúrgica de algunos ejemplares. El objetivo de este estudio fue evaluar diferentes parámetros espermáticos e histológicos, relacionados con la calidad seminal epididimal de hipopótamos comunes ubicados en el Magdalena Medio, Colombia. Dos hipopótamos fueron orquiectomizados quirúrgicamente y los espermatozoides fueron recuperados por los métodos combinados de lavado retrógrado e inyección de medio. Se realizó la evaluación de la movilidad (sistema SCA ${ }^{\circledR}$ ), la vitalidad espermática y la morfología espermática (prueba supravital), el potencial de membrana mitocondrial (JC-1), la integridad acrosómica (FITC-PNA), y la integridad estructural (SYBR14/IP) y funcional (prueba HOS) de la membrana plasmática. Asimismo, se realizó un análisis histológico de testículos y epidídimos. A excepción de la movilidad progresiva, la integridad funcional de membrana y la morfología espermática, se hallaron resultados muy similares para los parámetros de calidad seminal de ambos individuos. Las anormalidades espermáticas predominantes fueron gota citoplasmática y cola enrollada. El análisis histológico evidenció un proceso activo de espermatogénesis. Se concluye que la extracción epididimal de espermatozoides, posterior a la orquiectomía de hipopótamos comunes ubicados en un hábitat no nativo, permite obtener muestras espermáticas de buena calidad.

Palabras clave: Colombia, cinética espermática, histología.
Restrepo, G., Varela, E. \& Usuga, A. 2016. Evaluation of epididymal spermatic quality of hippopotamus Hippopotamus amphibius (Artiodactyla: Hippopotamidae) located in the Colombian Middle Magdalena. Acta Zoológica Mexicana (n. s.), 32(2): 158-167.

ABSTRACT. Common hippopotamus (Hippopotamus amphibius) is a mammal of the Artiodactyla order, living in Africa, with a declining population globally. With genetic conservation purposes, there have been processes of reproductive biotechnology, among which it is reported, recovery of sperm by methods as electroejaculation and extraction from the epididymis. This mammal is considered an exotic and invasive species was introduced in Colombia 1985; currently it reproduces out of control in the Middle Magdalena region. As a means of population control, it has performed the surgical sterilization of some specimens. The aim of this study was to evaluate different sperm and histological parameters related with epididymal semen quality of common hippopotamus located in the Colombian Middle Magdalena. Two hippopotamus were surgically orchiectomized and sperm were recovered by the combined methods of retrograde washing and medium injection. It was evaluated the sperm motility (SCA ${ }^{\circledR}$ system), the sperm vitality and morphology (supravital test), the mitochondrial membrane potential (JC-1), the acrosomal integrity (FITC-PNA), and the structural (SYBR14 / IP) and functional (HOS test) plasmatic membrane integrity. Also histological analysis of testis and epididymis were performed. Except for progressive motility, functional membrane integrity and sperm morphology, very similar results for semen quality parameters for both individuals were found. The predominant sperm abnormalities were cytoplasmic droplet and coiled tail. Histological analysis showed an active spermatogenesis process. We conclude that, after orchiectomy in common hippopotamus located in a non-native habitat, epididymal sperm extraction allows to obtain good quality sperm samples.

Key words: Colombia, sperm kinetics, histology. 


\section{INTRODUCCIÓN}

El hipopótamo común (Hippopotamus amphibius, Linneo 1758), es un mamífero del orden Artiodactyla, perteneciente a la familia: Hippopotamidae, que habita en África (Estes 1992). Está estrechamente relacionado con el hipopótamo pigmeo (Choeropsis liberiensis) (Eltringham 1993) y éste con los cetáceos, bóvidos y familias de los suidos (Ursing y Arnason 1998). Esta especie se extinguió en Argelia, Djibouti, Egipto y posiblemente en Liberia y Mauritania (Nowak 1991). Y de acuerdo con la Unión Internacional de Conservación de la Naturaleza (IUCN 2015), la población del hipopótamo común y el hipopótamo pigmeo (Choeropsis liberiensis), es considerada como vulnerable (Lewison \& Oliver 2008).

Los estudios recientes en esta especie se han centrado en su arquitectura anatómica (Fisher et al. 2010; Yoshitomi et al. 2012), etología (Blowers et al. 2010), fisiología (Noirard et al. 2008) y ecología (Cerling et al. 2008). En cuanto a su reproducción, se reporta en las hembras el estudio de los patrones endocrinos asociados a la ovulación y la preñez (Graham et al. 2002). Otros estudios han abordado la fisiología reproductiva del macho (Skinner et al. 1975; Erken et al. 1994; Kayanja 1989; Zapico 1999).

El macho no posee saco escrotal, sus testículos están envueltos por un tejido graso, tienen un cordón espermático corto y se encuentran localizados ventralmente al canal inguinal (Erken et al. 1994; Kayanja 1989). La masa de ambos testículos es de aproximadamente $266 \mathrm{~g}$ al inicio de la pubertad y $650 \mathrm{~g}$ en animales adultos (Smuts \& Whyte 1981). En los hipopótamos machos la espermatogénesis inicia a los dos años de edad, la pubertad se extiende entre los dos y los cinco años, y la madurez sexual se alcanza a los seis años. A nivel histológico, el diámetro promedio de los túbulos seminíferos es de $160 \mu$ m (Smuts \& Whyte 1981).

Este mamífero fue introducido en Colombia en 1985 y actualmente se le considera una especie invasora exótica que se distribuye y reproduce en la región del Magdalena Medio. En el 2014, se estimó una existencia de 60 ejemplares en el país, siendo considerados como invasores que amenazan con poner en peligro a las especies nativas, modificar el hábitat y alterar los procesos del ecosistema (Monsalve 2014). Dado lo anterior, en Colombia se decidió tomar medidas de control poblacional, mediante la esterilización quirúrgica de algunos ejemplares de la población censada cautiva. Como antecedente, se reportan dificultades intraquirúrgicas en la localización de los testículos y en el manejo de la anestesia (Erken et al. 1994;
Ramsay et al. 1998). Un estudio reciente describe el uso de ultrasonografía para la localización testicular, como una herramienta que facilita el procedimiento quirúrgico (Walzer et al. 2014).

Con fines de conservación genética de esta especie, se han realizado procesos de colecta, evaluación y criopreservación seminal. En un estudio de Saragusty et al. (2010a), se realizó la colecta de semen mediante la técnica de electroeyaculación. Se evaluaron los parámetros de calidad seminal: concentración, volumen, movilidad total, acrosomas intactos, morfología normal y la prueba hipoosmótica (HOS). Asimismo, se evaluaron diferentes diluyentes para la criopreservación de espermatozoides. Otro estudio reportó la recuperación de espermatozoides desde los epidídimos de hipopótamos comunes castrados, así como su criopreservación (Saragusty et al. 2010b).

El objetivo de este estudio fue evaluar diferentes parámetros espermáticos e histológicos, relacionados con la calidad seminal epididimal de hipopótamos comunes ubicados en el Magdalena Medio, Colombia. Lo anterior, como contribución al estudio de la biología reproductiva de esta especie en un hábitat no nativo, sin pretender fomentar la reproducción de la misma en ecosistemas donde sea considerada como invasora.

\section{MATERIALES Y MÉTODOS}

Orquiectomía. La orquiectomía se realizó de manera consecutiva en dos hipopótamos machos (Hippopotamus amphibius), ubicados en Puerto Triunfo (Antioquia, Colombia), de una edad aproximada entre cuatro y cinco años, con 800 y $1200 \mathrm{~kg}$ de peso. La anestesia se llevó a cabo según la técnica reportada por Walzer et al. (2014), con algunas modificaciones. Se usaron dardos con una solución compuesta de $1 \mathrm{mg} / \mathrm{kg}$ de ketamina (Imalgene $1000^{\circledR}$, Merial), $0.01 \mathrm{mg} / \mathrm{kg}$ de detomidina (Dormosedan $^{\circledR}$, Pfizer) y $0.05 \mathrm{mg} / \mathrm{kg}$ de butorfanol (Butormin ${ }^{\circledR}$, Holliday) (Belda et al., 2005). Posterior al derribo, las dosis se ajustaron por inyección directa y se emplearon tubos orotraqueales de $22 \mathrm{~mm}$ con neumotaponamiento.

Los animales se conectaron a una máquina de anestesia con $2 \%$ de isofluorano (Isoflurano ${ }^{\circledR}$, Piramal Healthcare UK Ltd.) para el mantenimiento de la anestesia (Laredo et al., 2001), se suministraron fluidos intravenosos y se realizó monitoreo de parámetros vitales durante todo el procedimiento. Se posicionaron en decúbito dorsal para permitir la incisión en la región paraprepucial. Se realizaron ligaduras con materiales absorbibles y puntos simples 
en piel con suturas no absorbibles. Para la reversión de la anestesia se aplicó $0.075 \mathrm{mg} / \mathrm{kg}$ de yohimbina (Yobine $^{\circledR}$, Lloyd Inc) y se medicaron con penicilina (Benzetacil LA $^{\circledR}$, Novartis) y ketoprofeno (Ankofen $10 \%{ }^{\circledR}$, Kyrovet). Extracción epididimal de espermatozoides. Los testículos fueron sumergidos en solución salina $(0.9 \% \mathrm{NaCl})$ y se colocaron en una caja de espuma de poliestireno en condiciones de refrigeración $\left(4-7^{\circ} \mathrm{C}\right)$ para ser transportados (Saragusty et al. 2010b). En el laboratorio, se recuperaron los espermatozoides provenientes del epidídimo. Para esto se realizó con una hoja de bisturí, la separación de cada epidídimo de su respectivo testículo (Fig. 1). Se eliminaron los tejidos periféricos de cada epidídimo y se procedió a colocarlo en posición vertical. Se canalizó el conducto deferente con una aguja calibre 18 y se realizó la extracción de los espermatozoides, por el método del

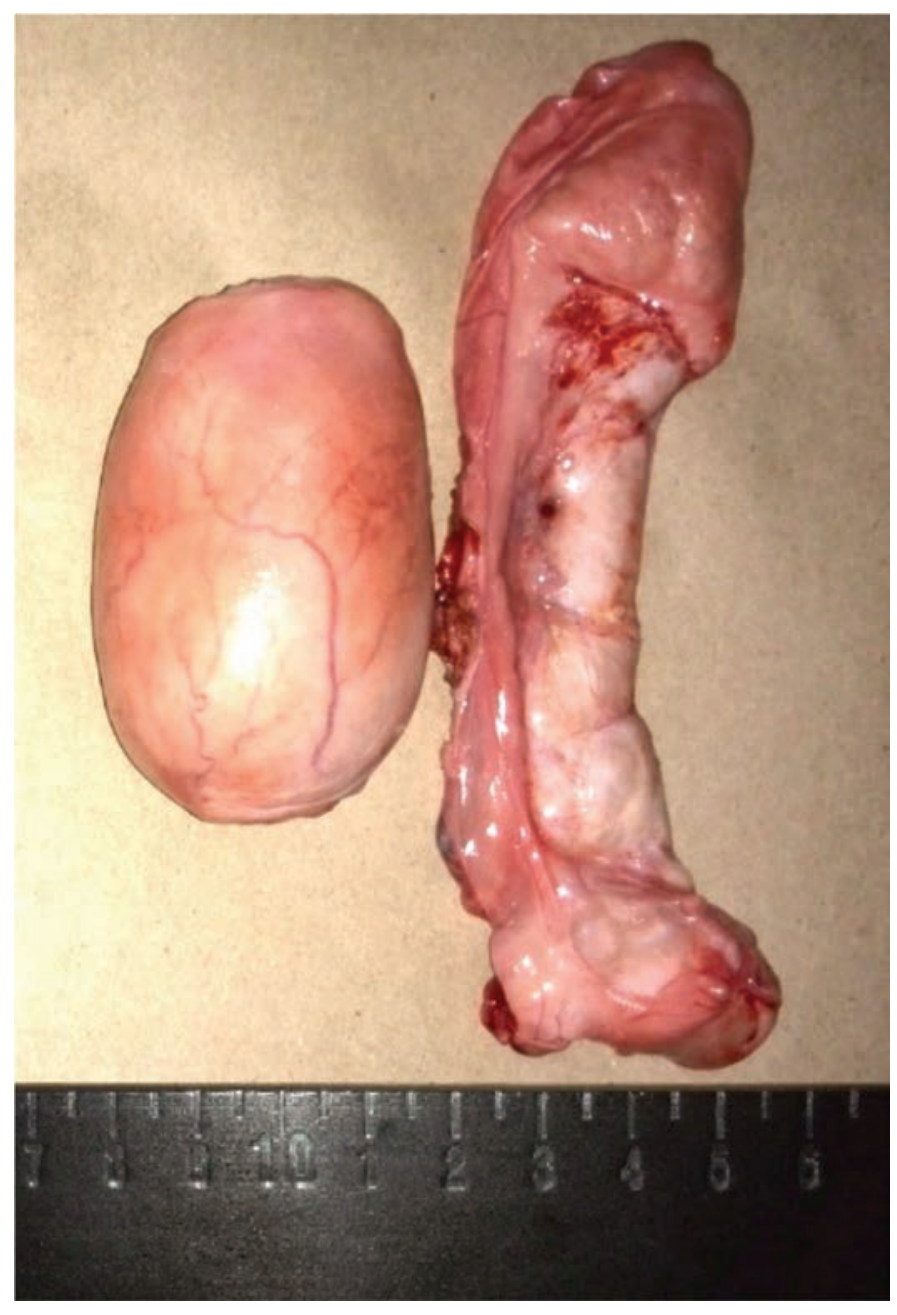

Figura 1. Testículo (izq.) y epidídimo (der.) de hipopótamo común. lavado retrógrado (Cary et al. 2004). A través del conducto y la cola del epidídimo, se aplicaron al menos 20 $\mathrm{mL}$ de diluyente (Equiplus, Minitube) precalentado a 37 ${ }^{\circ} \mathrm{C}$, el cual fue recolectado en un tubo estéril (Saragusty et al. 2010b). Este procedimiento se combinó con un método alternativo (inyección de medio), en el cual se inyectó diluyente a través de la pared del órgano y se realizaron cortes en toda su extensión. El fluido resultante se recolectó en una caja de Petri estéril.

Evaluación espermática. Movilidad espermática. Se empleó un procedimiento modificado del reportado por Hidalgo et al. (2005). Se colocó sobre un portaobjetos, una gota de $7.0 \mu \mathrm{L}$ del diluyente recuperado del lavado de los epidídimos. Se realizó la observación en un microscopio de contraste de fase (Eclipse E200, Nikon, Inc), con una cámara digital adaptada (Scout SCA780, Basler). La imagen fue procesada en un computador con el software Sperm Class Analizer (SCA ${ }^{\circledR}$, Microptic S.L.), mediante el cual se evaluaron la movilidad total (MT), la movilidad progresiva (MP), la velocidad curvilínea (VCL), la velocidad lineal (VSL), la velocidad media (VAP), los índice espermáticos de linealidad (LIN), rectitud (STR) y oscilación (IO), la amplitud del desplazamiento lateral de la cabeza (ALH), la frecuencia de batido de la cola (BCF), el área de la cabeza (AC), la hiperactividad (HA) y los recorridos circulares (RC). De igual forma, se realizó la agrupación de los espermatozoides por la velocidad de desplazamiento y por la relación entre velocidad y progresividad.

Integridad estructural de la membrana plasmática (IEM). Se evaluó mediante microscopía de fluorescencia, utilizando el procedimiento descrito por Gamboa et al. (2010), con el kit Live/Dead (Molecular Probes Inc). Se suspendieron $200 \mu \mathrm{L}$ de la muestra espermática, en solución Hanks Heppes $(\mathrm{HH})$ con $1 \%$ de albúmina sérica bovina (BSA), para una concentración aproximada de $20 \times 10^{6}$ espermatozoides / mL. Luego la mezcla fue incubada a 37 ${ }^{\circ} \mathrm{C}$ por 8 minutos, con $6 \mathrm{mM}$ de SYBR14. Seguidamente se incubó de la misma manera, con $0.48 \mathrm{mM}$ de yoduro de propidio. Luego a partir de una muestra de $5 \mu \mathrm{L}$, se realizó el conteo de 200 espermatozoides, mediante un filtro UV2A de un microscopio E200 con fluorescencia HBO (Nikon Inc.). Finalmente se estableció el porcentaje de espermatozoides con membrana íntegra (fluorescencia verde). Vitalidad espermática. Se evaluó mediante la prueba supravital con eosina-nigrosina (VE) (Barth y Oko 1989). Sobre un portaobjetos se depositó una gota de muestra y una gota de eosina-nigrosina (Sigma-Aldrich). Ambas gotas se mezclaron y se realizó un extendido, el cual fue 
fijado sobre una placa térmica a $37^{\circ} \mathrm{C}$. En un microscopio de contraste de fase (Eclipse E200, Nikon Inc.), se realizó el conteo de 200 espermatozoides y se calculó el porcentaje de espermatozoides vivos (no teñidos).

Morfología espermática. La morfología se evaluó a través de la técnica del test supravital con eosina-nigrosina, descrita previamente. En un microscopio de contraste de fase (Eclipse E200, Nikon Inc.), se evaluó la morfología individual de 200 espermatozoides. Se halló el porcentaje de espermatozoides con morfología anormal (MA), así como de las principales anormalidades observadas.

\section{Potencial de membrana interna mitocondrial (PMM).}

Se realizó el análisis de acuerdo con el protocolo descrito por Gravance et al. (2000). La muestra espermática se diluyó hasta $20 \times 10^{6}$ espermatozoides / $\mathrm{mL}$, y luego se incubó a $37^{\circ} \mathrm{C}$ por 8 minutos, con $2 \mathrm{mM}$ de yoduro de 5,5',6,6'-tetracloro-1,1',3,3' tetraetil benzimidazolil carbocianina (JC-1, Molecular Probes) en dimetilsulfóxido (DMSO). La evaluación se realizó usando un filtro B-2A de un microscopio E200 con fluorescencia HBO (Nikon Inc.). Se calculó el porcentaje de espermatozoides con fluorescencia roja (altos potenciales de membrana mitocondrial).

Integridad acrosómica (IA). Se evaluó mediante el uso de la sonda fluorescente FITC-PNA (Sigma), de acuerdo con lo descrito por Rathi et al. (2001). Se realizó un extendido de cada muestra espermática, el cual fue fijado por 10 minutos con etanol al 95\%. Luego, se dejó secar a temperatura ambiente y se agregaron $25 \mu \mathrm{L}$ de $5 \mu \mathrm{g} / \mathrm{mL}$ de FITC-PNA en solución buffer fosfato (PBS). Luego, cada extendido se guardó en la oscuridad por 30 minutos y fue lavado con agua destilada. Se evaluaron 200 espermatozoides usando un filtro G-2A, de un microscopio E200 con fluorescencia HBO (Nikon Inc.). Se halló el porcentaje de espermatozoides con acrosoma intacto (IA).

Integridad funcional de la membrana plasmática (HOS). Se empleó la metodología de la prueba hipoosmótica (Neild et al. 1999). En un tubo fueron mezclados $100 \mu \mathrm{L}$ de muestra espermática con $500 \mu \mathrm{L}$ de una solución hipoosmótica de sacarosa 5.4\% (100 mOsmol/L). La mezcla se incubó a $38.5^{\circ} \mathrm{C}$ durante 30 minutos. Posteriormente, en un microscopio de contraste de fase (Eclipse E200, Nikon Inc.), se evaluó la reacción (enrollamiento de la cola) de 200 espermatozoides, en mínimo cinco campos de observación diferentes. Se calculó el porcentaje de espermatozoides con membrana plasmática funcionalmente integra.

Análisis histológico. Se realizó el análisis histológico de muestras de testículo y epidídimo. Se obtuvieron seccio- nes de $1 \times 1 \mathrm{~cm}$ aproximadamente, las cuales se depositaron en formol neutro al $10 \%$ para su fijación, durante 24 horas. Luego, el formol fue reemplazado por alcohol al $70 \%$ y se realizaron cortes histológicos mediante un micrótomo. Las muestras fueron sometidas a coloración con Hematoxilina-Eosina (Álvarez et al. 2014). Posteriormente se analizaron mediante microscopía óptica y se obtuvieron imágenes de los cortes histológicos mediante una cámara Nikon DS-Fi2. Las imágenes fueron procesadas con el software NIS-Elements (Nikon Inc.).

\section{RESULTADOS}

Los resultados del análisis de calidad espermática, a partir de las muestras recuperadas de dos hipopótamos comunes, se presentan en el Cuadro 1. Los parámetros de movilidad y cinética espermática fueron bastante similares entre ambos individuos. Sin embargo, aunque el primer animal

Cuadro 1. Evaluación de la calidad espermática epididimal.

\begin{tabular}{lcc}
\hline Parámetro & Animal 1 & Animal 2 \\
\hline MT $(\%)$ & 59,9 & 47,6 \\
MP $(\%)$ & 52,6 & 36,3 \\
VCL $(\mu \mathrm{m} / \mathrm{s})$ & 111,1 & 113,3 \\
VSL $(\mu \mathrm{m} / \mathrm{s})$ & 26,7 & 34,2 \\
VAP $(\mu \mathrm{m} / \mathrm{s})$ & 81,9 & 91,9 \\
LIN $(\%)$ & 24,7 & 30,2 \\
STR $(\%)$ & 33,2 & 37,2 \\
IO (\%) & 73,9 & 81,1 \\
ALH $(\mu \mathrm{m} / \mathrm{s})$ & 2,2 & 2,2 \\
BCF (Hz) & 5,2 & 7,9 \\
AC $\left(\mu \mathrm{m}^{2}\right)$ & 26,2 & 24,7 \\
HA $(\%)$ & 17,1 & 12,9 \\
RC $(\%)$ & 48,3 & 35,3 \\
VE (\%) & 88,0 & 85,0 \\
IEM (\%) & 84,3 & 95 \\
PMM (\%) & 82,3 & 90,0 \\
IA (\%) & 94,0 & 88,0 \\
HOS (\%) & 65,0 & 36,0 \\
\hline
\end{tabular}

MT: movilidad total; MP: movilidad progresiva; VCL: velocidad curvilínea; VSL: velocidad lineal; VAP: velocidad media; LIN: índice de linealidad; STR: Índice de rectitud; IO: Índice de oscilación; ALH: amplitud de desplazamiento lateral de la cabeza; BCF: frecuencia de batido de la cola; AC: área de la cabeza; HA: hiperactividad; RC: recorridos circulares; VE: vitalidad espermática; IEM: integridad estructural de membrana; PMM: potencial de membrana mitocondrial; IA: integridad acrosómica; HOS: integridad funcional de membrana plasmática. 
presentó mayores valores de movilidad (MT y MP), los diferentes parámetros de cinética espermática incluyendo los índices de linealidad (LIN), oscilación (IO) y rectitud (STR), fueron superiores para el segundo individuo. Otros parámetros en que se observaron diferencias marcadas entre ambos machos, fueron los recorridos circulares (RC) y los indicadores de integridad estructural (IEM) y funcional de la membrana plasmática (HOS).

La evaluación morfológica evidenció altos porcentajes de anormalidades espermáticas para ambos individuos. En el Cuadro 2 se presenta la distribución de los principales defectos morfológicos observados. La alta proporción de gotas citoplasmáticas proximales en ambos individuos, así como la predominancia de células con cola enrollada en el segundo macho, representaron las principales anormalidades espermáticas encontradas.

El análisis individual de la cinética de los espermatozoides de ambos hipopótamos, con base a la velocidad de desplazamiento, se presenta en la figura 2 . Los resul-
Cuadro 2. Evaluación morfológica de los espermatozoides.

\begin{tabular}{lcr}
\hline Morfología & Animal 1 & Animal 2 \\
\hline Gota citoplasmática proximal (\%) & 42.0 & 23.0 \\
Cabeza desprendida (\%) & 6.0 & 1.0 \\
Cola enrollada (\%) & 5.0 & 61.0 \\
Doble Cabeza (\%) & 1.0 & 0 \\
Microcefalias (\%) & 1.0 & 0 \\
Morfología Anormal (\%) & 55 & 85 \\
\hline
\end{tabular}

tados indican predominancia de espermatozoides rápidos e inmóviles para ambos individuos, sin embargo, para el segundo individuo más del 50\% de toda la población espermática recuperada y evaluada correspondió a células inmóviles. La figura 3 presenta el análisis cinético para la agrupación entre la velocidad y la progresividad. Entre las células móviles, se encontró predominio de espermatozoides medios progresivos para ambos individuos. Sin embargo, acorde con el análisis de velocidad espermática

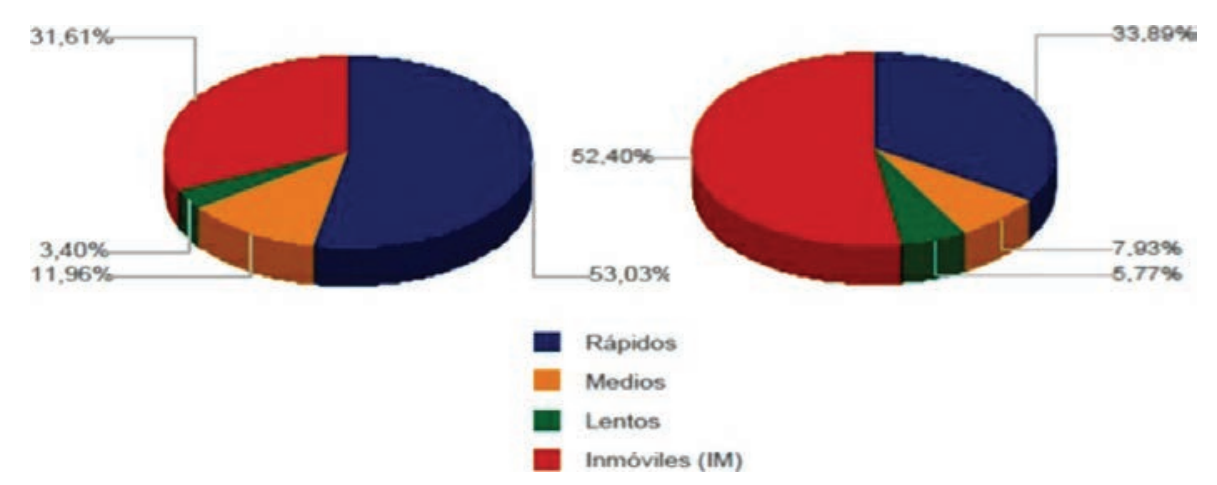

Figura 2. Velocidad espermática. Animal 1 (izq.), Animal 2 (der.). Lentos: VCL $<20 \mu \mathrm{m} / \mathrm{s}$. Medios: VCL 20-45 $\mu \mathrm{m} / \mathrm{s}$. Rápidos VCL 46-90 $\mu \mathrm{m} / \mathrm{s}$.

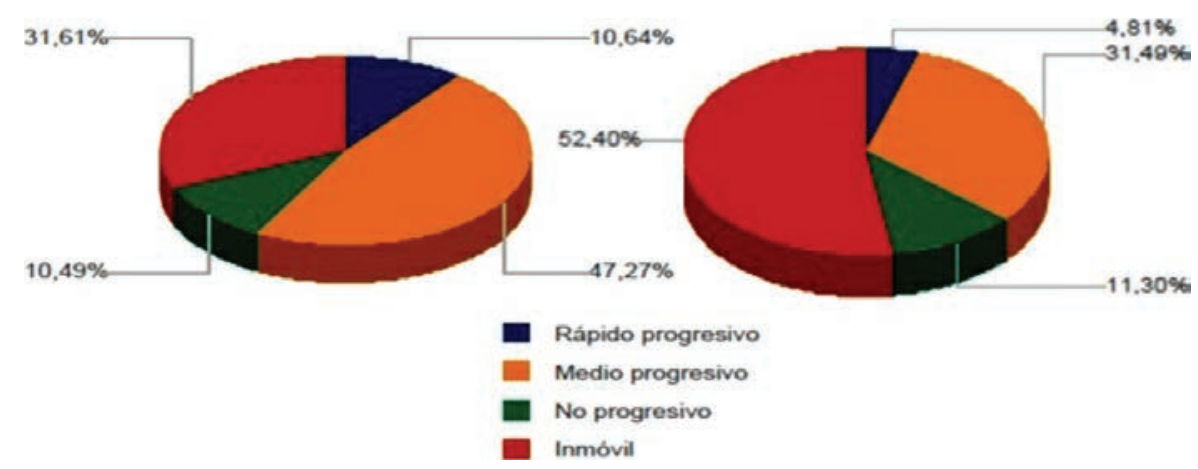

Figura 3. Velocidad y progresividad espermática. Animal 1 (izq.), Animal 2 (der.). Lentos: VCL < $20 \mu \mathrm{m} / \mathrm{s}$. Medios: VCL 20-45 $\mu \mathrm{m} / \mathrm{s}$. Rápidos: VCL $46-90 \mu \mathrm{m} / \mathrm{s}$. Progresivos: STR > 75\%. 

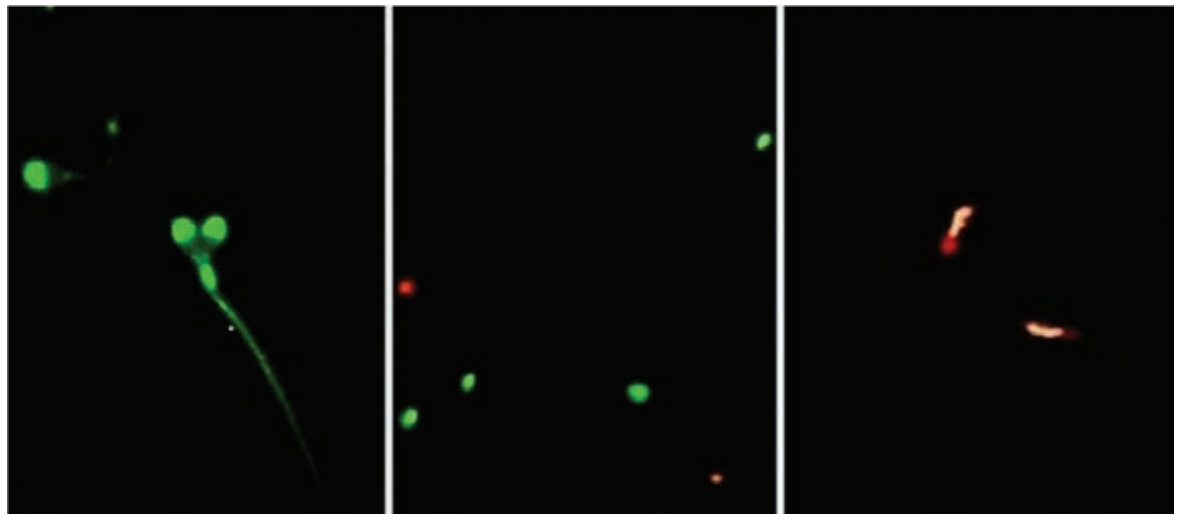

Figura 4. Evaluación de espermatozoides por fluorescencia. Integridad del acrosoma FITC-PNA (izquierda), Integridad estructural de membrana SYBR14/IP (medio), Potencial de membrana mitocondrial JC-1 (Derecha).

(Fig. 2), existió una alta proporción de espermatozoides inmóviles, la cual fue mayor para el segundo macho.

El análisis de la calidad seminal de ambos machos mediante sondas fluorescentes mostró la funcionalidad de los métodos empleados en las células espermáticas de la especie. La figura 4 presenta ejemplos de la evaluación seminal realizada por microscopía de fluorescencia para los parámetros de IEM, IA y PMM.

La evaluación histológica de las muestras obtenidas a partir del tejido testicular y epididimal, permitió la caracterización estructural y celular de los tejidos, así como la actividad espermatogénica en desarrollo en ambos machos. En la figura 5 se presenta un corte histológico

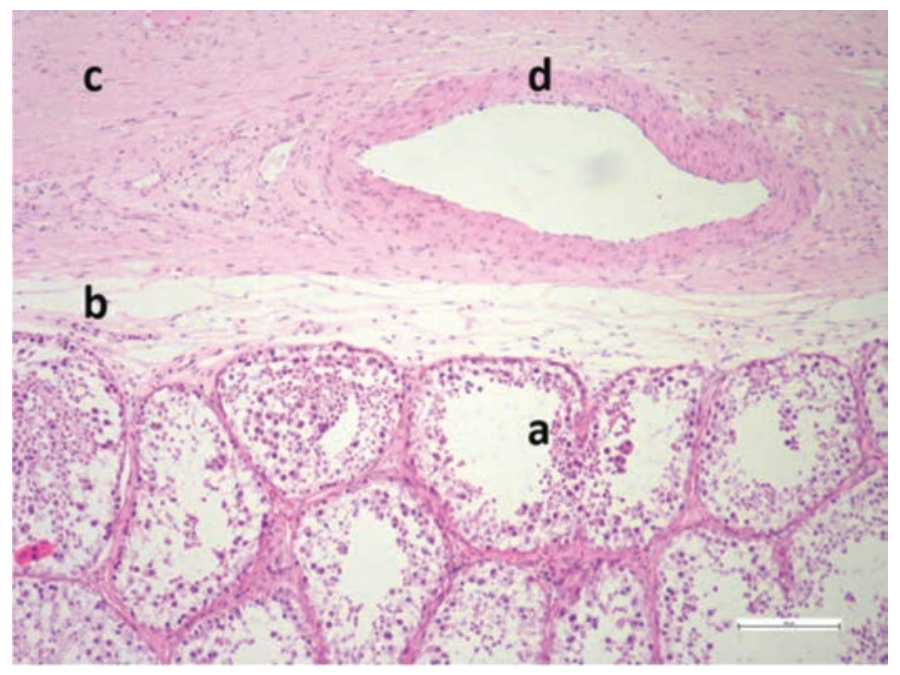

Figura 5. Tejido testicular. Túbulos seminíferos (a), tejido conectivo (b), túnica albugínea (c) y arteria (d). de tejido testicular, en el cual se observan varios túbulos seminíferos con poca celularidad, tejido conectivo y la túnica albugínea, en la que se encontraron diversos vasos sanguíneos.

En la figura 6 se observa el túbulo seminífero rodeado por las células de la lámina basal, las cuales presentan un aspecto alargado, rodeadas a su vez por músculo liso. Se identifican células sexuales masculinas en diferentes estadios. Además, se observan las células de Leydig en el intersticio tubular. En algunos cortes histológicos se observó una disminución en la población celular del túbulo seminífero, posiblemente por la edad temprana de los animales. En la figura 7 se observan espermatozoides

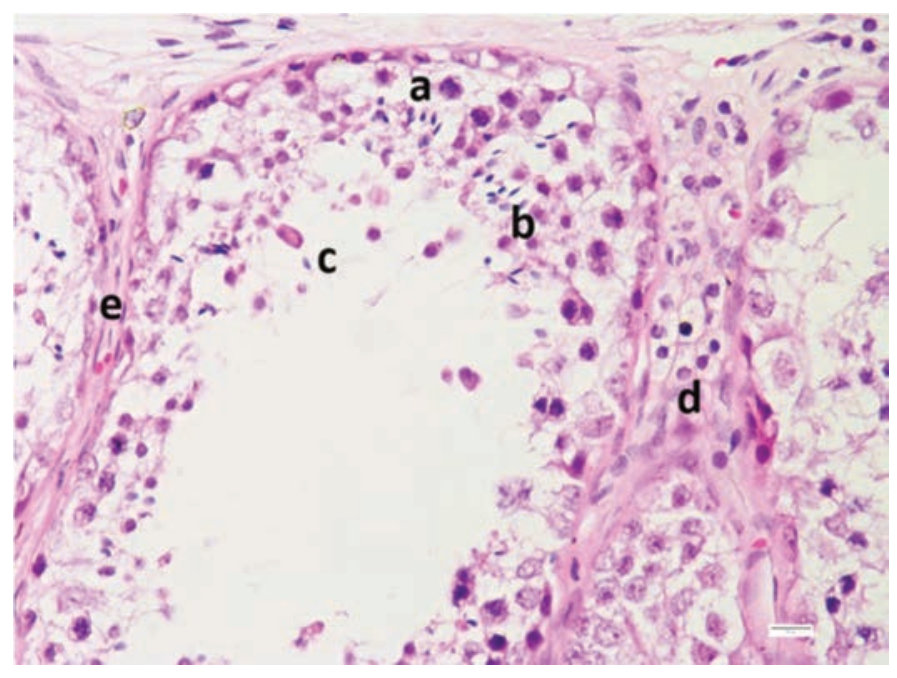

Figura 6. Túbulo seminífero. Espermatogonias (a), espermátides (b), espermatozoides (c), células de Leydig (d) y lámina basal (e). 


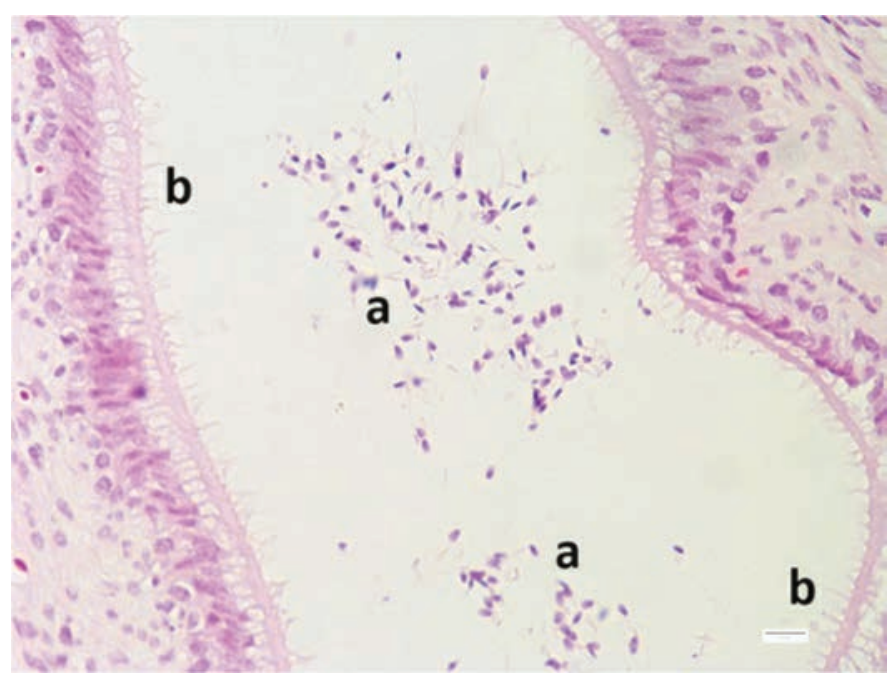

Figura 7. Cola del epidídimo. Espermatozoides (a), epitelio cilíndrico pseudoestratificado (b).

totalmente desarrollados en la luz de la cola del epidídimo y se identifica el epitelio cilíndrico pseudo estratificado con estereocilios.

\section{DISCUSIÓN}

Aún son pocos los estudios en que se reporta la evaluación espermática en hipopótamos. En un estudio con el hipopótamo pigmeo (Choeropsis liberiensis), a partir de muestras de semen obtenidas por electro-eyaculación, se encontraron valores promedio para MT, IA, MA y HOS, de $75.7 \% \pm 6.9 \% ; 87.8 \% \pm 1.2 \%$; $52.7 \% \pm 4.3 \%$ y $79.3 \%$ $\pm 4.4 \%$, respectivamente (Saragusty et al. 2010a).

La recuperación exitosa de espermatozoides directamente desde los epidídimos ha sido realizada en diferentes especies de mamíferos, en los que se destacan bovinos, equinos y caninos (Turri et al. 2012; Olaciregui et al. 2014, Mota et al. 2014). Para el caso del hipopótamo común, un estudio reportó la extracción de espermatozoides desde la cola del epidídimo y su posterior evaluación, con resultados para MT, IA, MA y HOS de $88.1 \pm 4.2 \%$; $87.7 \pm 1.8 \%$; $51.6 \pm 4.1 \%$ y $83.3 \pm 1.8 \%$, respectivamente (Saragusty et al. 2010b). Estos resultados fueron superiores para MT y HOS, con respecto a los obtenidos en el presente estudio, mientras para IA y MA, podrían asumirse como similares (Cuadros 1 y 2). Sin embargo, es importante considerar que factores como el método de recolección, el diluyente utilizado, el efecto individual del animal y la edad, podrían ser fuente de variación en los resultados.

No se encontraron reportes de evaluación de parámetros cinéticos del semen de hipopótamos. En comparación con una investigación donde se extrajeron espermatozoides de los epidídimos de porcinos (Sus scrofa domestica, Linneo 1758), también pertenecientes al orden Artiodactyla, se observan promedios similares a los encontrados en este estudio (Cuadro 1), para MT (52.5 $\pm 2.6 \%)$, LIN $(26.4 \pm 0.7 \%)$ y BCF $(4.9 \pm 0.2 \mathrm{~Hz})$; promedios inferiores para MP $(23.9 \pm 1.4 \%)$, VCL $(73.4 \pm 1.3 \mu \mathrm{m} / \mathrm{s})$, VSL (19.4 $\pm 0.7 \mu \mathrm{m} / \mathrm{s}), \operatorname{VAP}(34.5 \pm 0.7 \mu \mathrm{m} / \mathrm{s})$ e IO $(46.8 \pm 0.5) ; \mathrm{y}$ promedios superiores para STR $(56.1 \pm 1.0 \%)$ y ALH (3.8 $\pm 0.1 \mu \mathrm{m} / \mathrm{s}$ ) (Avilés 2011). Sin embargo, además de las probables diferencias existentes entre las especies, es importante considerar que la movilidad espermática depende de una multitud de factores pre-testiculares, testiculares y post-testiculares (Elzanaty et al. 2002). Adicionalmente, fue evidente el efecto individual del macho en la calidad seminal, a través de las diferencias encontradas en los resultados de movilidad y cinética espermática entre ambos animales (Cuadro 1).

La hiperactivación de los espermatozoides se relaciona con un aumento en la amplitud lateral de la cabeza, movilidad no progresiva y una baja frecuencia de batido de la cola. En humanos se han definido como espermatozoides hiperactivos, aquéllos con valores de VCL $>70$ $\mu \mathrm{rn} / \mathrm{s}$, ALH $\geq 7 \mu \mathrm{m}, \mathrm{LIN} \leq 30 \%$ y VSL $\leq 30 \mu \mathrm{m} / \mathrm{s}$ (Verstegen et al. 2002). En coincidencia con la mayoría de estos parámetros, los individuos evaluados en este trabajo presentaron una baja proporción de hiperactivación espermática (12.9\% y 17.1\%).

La evaluación de la vitalidad ha avanzado desde el uso de tinciones convencionales, hasta la aplicación de métodos fluorescentes. En espermatozoides de bovino (Bos spp., Lineo 1758) extraídos de la cola del epidídimo, se encontró un promedio de $69.4 \pm$ $10.7 \%$ para la vitalidad por eosina-nigrosina, y de 77.7 $\pm 10.7 \%$, cuando fue evaluada a través de sondas fluorescentes (Ribeiro-Peres et al. 2014). Para los animales evaluados en este estudio se encontraron valores superiores por ambos métodos expresados como VE e IEM (Cuadro 1).

A diferencia de la mayoría de los parámetros evaluados, se observó una marcada diferencia entre ambos individuos para la prueba HOS (Cuadro 1). Este método evalúa la integridad funcional de la membrana plasmática, siendo útil como indicador de la fertilidad potencial de los espermatozoides (Ramu \& Jeyendran 2013). En un 
estudio reciente con espermatozoides bovinos extraídos desde los epidídimos, se encontró un promedio para HOS de $69.7 \pm 9.2 \%$ (Ribeiro-Peres et al. 2014).

Para ambos individuos se hallaron altos porcentajes de PMM e IA (Cuadro 1). No se encontraron otros reportes de evaluación de estos parámetros en hipopótamos. Sin embargo, otros estudios reportan la evaluación del PMM y la IA en espermatozoides epididimales de Artiodáctilos, como el ciervo rojo (Cervus elaphus; Anel-López et al. 2015); el bison norteamericano (Bison bison Linneo, 1758; Krishnakumar et al. 2011) y el íbice ibérico (Capra pirenaica Schin, 1838; López-Saucedo et al. 2014).

El análisis de la distribución de los espermatozoides por su velocidad (Fig. 2), y por su velocidad y progresividad (Fig. 3), evidenció una alta proporción de espermatozoides inmóviles, así como predominancia de patrones de movimiento rápido y medio-progresivo. Es probable que factores como el origen epididimario, el método de extracción espermática y la dilución en un medio no especifico para la especie, pudiesen ser determinantes en estos resultados. De acuerdo con Goovaerts et al. (2006), los espermatozoides eyaculados difieren de los espermatozoides epididimales, en sus características de movimiento. Siendo estos últimos tendientes a poseer una menor fertilidad (Papa et al. 2015). Adicionalmente, Turri et al. (2012) encontraron que la técnica de extracción por flujo retrógrado es mejor que el método de flotación, en términos de calidad espermática, movilidad total y viabilidad.

La morfología espermática podría ser uno de los parámetros más afectados por la edad de los reproductores. Las anormalidades más frecuentes en bovinos jóvenes son las que se presentan en la pieza media, como gotas citoplasmáticas proximales, pieza intermedia reflejada distalmente y pieza intermedia inclinada (Menon et al. 2011). Para los individuos evaluados, la predominancia de gotas citoplasmáticas proximales (Cuadro 2), podría considerarse como producto de una espermatogénesis en proceso de maduración. Más aún cuando en promedio la madurez sexual en los hipopótamos se alcanza a los seis años de edad (Smuts y Whyte 1981).

A partir del análisis histológico se observaron túbulos seminíferos con poca celularidad, lo cual podría atribuirse a la edad temprana de los animales (Fig. 5). Smuts y Whyte (1981) reportaron la presencia de los primeros espermatozoides en los túbulos seminíferos de hipopótamos a los cinco años de edad; sin embargo, también observaron la presencia de pocos espermatozoides en los epidídimos de animales entre los dos y los cinco años de edad. Este fenómeno contradictorio fue explicado por estos autores, por la posible autólisis acelerada del tejido testicular postmortem.

A partir de este estudio, también se evidenció la ocurrencia de un proceso activo de espermatogénesis, ya que se identificaron células sexuales masculinas en diferentes estadios, como células germinales o espermatogonias, espermátides y espermatozoides (Fig. 6). Además del hallazgo de espermatozoides completamente desarrollados en la luz de la cola del epidídimo (Fig. 7). En contraste con otras especies de ungulados, con una edad a la pubertad más temprana, se reporta que, en toros de 54 semanas de edad, la mayoría de los túbulos seminíferos ya poseen células germinales avanzadas, como espermátides redondas y espermátides alargadas (Moura et al. 2011).

\section{CONCLUSIONES}

La extracción epididimal de espermatozoides posterior a la castración de hipopótamos comunes ubicados en un hábitat no nativo, permite la obtención de muestras espermáticas de buena calidad. No obstante la edad de los animales incluidos en el estudio, se observó un proceso de espermatogénesis activo y la presencia de células espermáticas viables, lo cual podría contribuir al conocimiento de la biología reproductiva y de la madurez sexual de ejemplares desarrollados en hábitats foráneos. Este estudio permitió la evaluación de diferentes parámetros espermáticos, así como una caracterización histológica testicular y epididimal de los cuales no se conocen reportes previos para la especie.

AGRADECIMIENTOS. Juan Pablo Villegas, Universidad CES, Medellín, Antioquia, Colombia. Parque Temático Hacienda Nápoles, Puerto Triunfo, Antioquia, Colombia.

\section{LITERATURA CITADA}

Álvarez, G., Rojano, C., Carrascal, J., Chacón, J. \& Martínez, C. 2014. Histopatología de órganos y lesiones en hicoteas Trachemys callirostris callirostris (Gray, 1856) mantenidas en cautiverio en Córdoba, Colombia. Revista CES Medicina Veterinaria y Zootecnia, 9: 15-25.

Anel-López, L., Martínez-Rodríguez, C., Soler, A., FernándezSantos, M., Gardea, J. \& Morrell, J. 2015. Use of Androcoll-S after thawing improves the quality of electroejaculated and epididymal sperm samples from red deer. Animal Reproduction Science, 158: 68-74.

Avilés, K. 2011. Estudio de la funcionalidad espermática, interacción de gametos y análisis proteico en espermatozoides epididimarios 
y eyaculados en la especie porcina. Tesis doctoral, Facultad de Veterinaria, Universidad de Murcia, Región de Murcia, 239 pp.

Barth, A. \& Oko, R. 1989. Anormal morfology of bovine spermatozoa. Iowa State University Press, 1ra ed. Iowa, USA, 285 pp.

Belda, E., Laredo, F. G., Escobar, M., Agut, A., Soler, M. \& Lucas, X. 2005. Agonistas $\alpha-2$ adrenérgicos en sedación y anestesia veterinaria. Anales de Veterinaria, 21: 23-33.

Blowers, T., Waterman, J., Kuhar, C. \& Bettinger, T. 2010. Social behaviors within a group of captive female Hippopotamus amphibius. Journal of Ethology, 28: 287-294.

Cary, J., Madill, S., Farnsworth, K., Hayna, J., Duoos, L. \& Fahning, M. 2004. A comparison of electroejaculation and epididymal sperm collection techniques in stallions. Canadian Veterinary Journal, 45: 35-41.

Cerling, T., Harris, J., Hart, J., Kaleme, P., Klingel, H., Leakey, M., Levin, N., Lewison, R. \& Passey, B. 2008. Stable isotope ecology of the common hippopotamus. Journal of Zoology, 276: 204-212.

Eltringham, S. 1993. The Pygmy Hippopotamus (Hexaprotodon liberiensis). Pp 55-60. In: W. Oliver (Ed.). Pigs, peccaries, and hippos. International Union for the Conservation of Nature, Gland, Switzerland.

Elzanaty, S., Richthoff, J., Malm, J. \& Giwercman, A. 2002. The impact of epididymal and accessory sex gland function on sperm motility. Human Reproduction, 17: 2904-2911.

Erken, A., Klaver, P. \& Frankenhuis, M. 1994. Castration and sterilization of an adult male hippopotamus. Erkrankungen der Zootiere. 36: 333-335.

Estes, R. 1992. The behavior guide to African mammals: including hoofed mammals, carnivores, primates, 20th Anniversary Edition. University of California Press, 1ra ed. Los Angeles, California.

Fisher, R., Scott, K. \& Adrian, B. 2010. Hind limb myology of the common hippopotamus, Hippopotamus amphibius (Artiodactyla: Hippopotamidae). Zoological Journal of the Linnean Society, 158: 661-682.

Gamboa, S., Rodrigues, A., Henriques, I., Batista, C. \& RamalhoSantos, J. 2010. Seasonal functional relevance of sperm characteristics in equine spermatozoa. Theriogenology, 73: 950-958.

Goovaerts, I., Hoflack, G., Van Soom, A., Dewulf, J., Nichi, M., de Kruif, A. \& Bols, P. 2006. Evaluation of epididymal semen quality using the Hamilton-Thorne analyzer indicates variation between the two caudae epididymides of the same bull. Theriogenology, 66: 323-330.

Graham, L., Reid, K., Webster, T., Richards, M. \& Joseph, S. 2002. Endocrine patterns associated with reproduction in the Nile hippopotamus (Hippopotamus amphibius) as assessed by fecal progestagen analysis. General and Comparative Endocrinology, 128: 74-81.

Gravance, C., Garner, D., Baumber, J. \& Ball, B. 2000. Assessment of equine sperm mitochondrial function using JC-1. Theriogenology, 53: 1691-1703.

Hidalgo, M., Rodriguez I, Dorado J, Sanz J, \& Soler C. 2005. Effect of sample size and staining methods on stallion sperm morphometry by the Sperm Class Analyzer. Vet Med - Czech 2005; 50: 24-32.

Kayanja, F. 1989. The reproductive biology of the male hippopotamus. Pp. 181-196. In: F. Kayanja, P. Jeweel \& G. Maloiy (Eds.).
Symposia of the Zoological Society of London, Oxford University Press, London, UK.

Krishnakumar, S., Whiteside, D., Elkin, B. \& Thundathil, J. 2011. Evaluation of an animal protein-free semen extender for cryopreservation of epididymal sperm from North American bison (Bison bison). Theriogenology, 76: 252-260.

Laredo, F., Gómez-Villamandos, R., Redondo, J. I., Cruz, J. I. \& Burzaco, O. 2001. Anestesia inhalatoria: bases, drogas y equipamiento. Consulta de Difusión Veterinaria, 9: 69-83.

Lewison, R. \& Oliver, W. (IUCN SSC Hippo Specialist Subgroup). 2008. Hippopotamus amphibius. The IUCN Red List of Threatened Species 2008: e.T10103A3163790.doi: 10.2305/IUCN. UK.2008.RLTS.T10103A3163790

López-Saucedo, J., Paramio, M., Fierro, R., Izquierdo, D., Catalá, M., Coloma, M., Toledano-Díaz, A., López-Sebastián, A. \& Santiago-Moreno, J. 2014. Sperm characteristics and heterologous in vitro fertilisation capacity of Iberian ibex (Capra pyrenaica) epididymal sperm, frozen in the presence of the enzymatic antioxidant catalase. Cryobiology, 68: 389-394.

Menon, A., Barkema, H., Wilde, R., Kastelic, J. \& Thundathil, J. 2011. Associations between sperm abnormalities, breed, age, and scrotal circumference in beef bulls. Canadian Journal of Veterinary Research, 75: 241-247.

Monsalve, S. 2014. Los hipopótamos (Hippopotamus amphibius) en Colombia. Especie exótica, introducida e invasora. Revista LasaIlista Investigación, 11: 229-230.

Mota, A., Rodrigues, H., Pereira, T., de Sousa, M., de Freitas, L. de Araújo A. \& da Silva, L. 2014. Cryopreservation of canine epididymal sperm using ACP-106c and TRIS. Cryobiology, 69: 17-21.

Moura, A., Souza, C. \& Erickson B. 2011. Early prepubertal testis criteria, seminiferous epithelium and hormone concentrations as related to testicular development in beef bulls. Animal Reproduction Science, 124: 39-47.

Neild, D., Chaves, G., Flores, M., Mora, N., Beconi, M. \& Agüero, A. 1999. Hypoosmotic test in equine spermatozoa. Theriogenology, 51: 721-727.

Noirard, C., Le Berre, M., Ramousse, R. \& Lena, J. 2008. Seasonal variation of thermoregulatory behavior in the Hippopotamus ( $\mathrm{Hi}$ ppopotamus amphibius). Journal of Ethology, 26: 191-193.

Nowak, R. 1991. Walker's Mammals of the World. The Johns Hopkins University Press, 5th ed. Baltimore, Maryland, EUA, 1629 pp.

Olaciregui, M., Gil, L., Montón, A., Luño, V., Jerez, R. \& Martí, J. 2014. Cryopreservation of epididymal stallion sperm. Cryobiology, 68: 91-95.

Papa, P., Papa, F., Oliveira, L., Guasti, P., Castilho, C. \& Giometti, I. 2015. Different extenders in the cryopreservation of bovine epididymal spermatozoa. Animal Reproduction Science, In Press.

Ramsay, E., Loomis, M., Mehren, K., Boardman, W., Jensen, J. \& Geiser, D. 1998. Chemical restraint of the Nile hippopotamus (Hippopotamus amphibius) in captivity. Journal of Zoo and Wildlife Medicine, 29: 45-49.

Ramu, S. \& Jeyendran, R. 2013. The hypo-osmotic swelling test for evaluation of sperm membrane integrity. Methods in Molecular Biology, 927: 21-25.

Rathi, R., Colembrander, B., Bevers, M. \& Gadella, B. 2001. Evaluation of in vitro capacitation of stallion spermatozoa. Biology of 
Reproduction, 65: 462-470.

Ribeiro-Peres, A., Munita-Barbosa, L., Yumi-Kanazawa, M., Mello-Martins, M. \& Ferreira de Souza, F. 2014. Criopreservación de espermatozoides bovinos extraídos de la cola del epidídimo utilizando los métodos convencional y automatizado. Archivos de Medicina Veterinaria, 46: 31-38.

Saragusty, J., Hildebrandt, T., Bouts, T., Göritz, F. \& Hermes, R. 2010a. Collection and preservation of pygmy hippopotamus (Choeropsis liberiensis) semen. Theriogenology, 74: 652-657.

Saragusty, J., Walzer, C., Petit, T., Stalder, G., Horowitz, I. \& Hermes, R. 2010b. Cooling and freezing of epididymal sperm in the common hippopotamus (Hippopotamus amphibius). Theriogenology, 74: 1256-1263.

Skinner, J., Scorer, J. \& Millar, R. 1975. Observations on the reproductive physiological status of mature herd bulls, bachelor bulls and young bulls in the hippopotamus Hippopotamus amphibius amphibius Linnaeus. General and Comparative Endocrinology, 26: 92-95.

Smuts, G., \& Whyte, I. 1981. Relationships between reproduction and environment in the hippopotamus Hippopotamus amphibius in the Kruger National Park. Koedoe, 24: 169-185.
Turri, F., Madeddu, M., Gliozzi, T., Gandini, G. \& Pizzi, F. 2012. Influence of recovery methods and extenders on bull epididymal spermatozoa quality. Reproduction in Domestic Animals, 47: 712717.

Ursing, B. \& Arnason, U. 1998. Analyses of mitochondrial genomes strongly support a hippopotamus-whale clade. Proceedings of the Royal Society of London B Biological Sciences, 265: 2251-2255.

Verstegen, J., Iguer-Ouada, M. \& Onclin, K. 2002. Computer assisted semen analyzers in andrology research and veterinary practice. Theriogenology, 57: 149-179.

Walzer, C., Petit, T., Stalder, G., Horowitz, I., Saragusty, J. \& Hermes, R. 2014. Surgical castration of the male common hippopotamus (Hippopotamus amphibius). Theriogenology, 81: 514-518.

Yoshitomi, S., Kawashima, T., Murakami, K., Takayanagi, M., Inoue, Y., Aoyagi, R. \& Sato, F. 2012. Anatomical architecture of the brachial plexus in the common hippopotamus (Hippopotamus amphibius) with special reference to the derivation and course of its unique branches. Anatomia Histologia Embryologia, 41: 280-285.

Zapico, T. 1999. First documentation of flehmen in a common hippopotamus (Hippopotamus amphibius). Zoo Biology, 18: 415-420. 